\title{
Implante de Stent Farmacológico para o Tratamento da Reestenose de Outro Stent Farmacológico: Análise Tardia do Registro DESIRE
}

\author{
José de Ribamar Costa Jr. ${ }^{1}$, Amanda G. M. R. Sousa ${ }^{1}$, Adriana C. Moreira', Ricardo Alves da Costa ${ }^{1}$, \\ Galo Maldonado ${ }^{1}$, Manuel N. Gano', Ibraim Pinto ${ }^{1}$, Cantídio Campos ${ }^{1}$, \\ Rodolfo Staico ${ }^{1}$, Maria Helena Dib¹, Ricardo Pavanello' ${ }^{1}$, J. Eduardo Sousa ${ }^{1}$
}

\section{RESUMO}

Fundamentos: Apesar da notória redução nas taxas de reestenose (RIS) com os stents farmacológicos (SF), essa complicação ainda ocorre em $5 \%$ a $25 \%$ dos casos. A história natural e o melhor tratamento para a reestenose de SF ainda não estão definidos. Método: Entre maio de 2002 e novembro de 2007, todos os pacientes com RIS de SF tratados com outro SF foram consecutivamente incluídos neste registro. O tipo de SF implantado para tratar a RIS ficou a critério do operador. Acompanhamento clínico foi obtido com 1, 6 e 12 meses e, então, anualmente. O objetivo primário foi avaliar a incidência acumulada de eventos cardíacos maiores (ECAM). Resultados: Dos 45 pacientes (53 lesões) tratados percutaneamente com outro SF, $66,6 \%$ eram do sexo masculino. Diabetes melito foi observado em $35,5 \%$ desses pacientes. A maioria das reestenoses foi do tipo focal $(75 \%)$. O diâmetro de referência do vaso tratado e a extensão da lesão foram de 2,99 \pm $0,5 \mathrm{~mm}$ e 11,33 $\pm 5,8 \mathrm{~mm}$, respectivamente. Em $28 \%$ dos casos, optou-se por implantar um SF diferente daquele que apresentou RIS. Sucesso angiográfico foi obtido em $100 \%$ dos casos. Seguimento clínico foi obtido em toda a população (média de $2,7 \pm 1,1$ anos). No seguimento tardio, a taxa cumulativa de ECAM foi de $13,3 \%$, com 5 $(11,1 \%)$ pacientes manifestando recorrência de sintomas. Conclusões: Nesta série, o uso de outro SF para tratar uma RIS de SF mostrou-se factível, seguro e eficaz, com baixas taxas de eventos adversos a curto e longo prazos.

DESCRITORES: Endoprótese, efeitos adversos. Reestenose coronária. Angioplastia transluminal percutânea coronária. Coronariopatia. Resultado de tratamento.

1 Instituto de Ensino e Pesquisa - Hospital do Coração - Associação do Sanatório Sírio - São Paulo, SP.

Correspondência: J. Eduardo Sousa. Rua Desembargador Eliseu Guilherme, 147 - Paraíso - São Paulo, SP - CEP 04004-030

E-mail: jesousa@uol.com.br

Recebido em: 5/6/2008 • Aceito em: 26/8/2008

\section{SUMMARY}

Drug-Eluting Stent Implantation for the Treatment of Drug-Eluting Stent Restenosis: Long-Term Analysis of the DESIRE Registry

Background: Despite the marked reduction in restenosis rates following drug-eluting stent (DES) use, this complication still happens in 5\%-25\% of the cases. The natural history and the best treatment for DES in-stent restenosis are still to be defined. Methods: Between May 2002 and November 2007, all consecutive cases of DES restenosis treated with another DES were included in this registry. The type of DES to be deployed was at the surgeon's discretion. Follow-up data were obtained at 1,6 , and 12 months and then annually. The primary endpoint of this study was the incidence of combined major cardiac events (MACE). Results: Of the 45 patients (53 lesions) submitted to new percutaneous coronary intervention $(\mathrm{PCI})$ with $\mathrm{DES}, 66.6 \%$ were men. Diabetes mellitus was observed in $35.5 \%$ of the patients. Most in-stent restenosis were focal (75\%). Reference vessel diameter and lesion length were $2.99 \pm 0.5 \mathrm{~mm}$ and 11.33 $\pm 5.8 \mathrm{~mm}$, respectively. A different type of DES was preferred in $28 \%$ of the cases. Angiographic success was achieved in all cases. Clinical data of all population were obtained (mean $2.7 \pm 1.1$ years). In the long term, the cumulative rate of MACE was $13.3 \%$, and $5(11.1 \%)$ patients manifested recurrent symptoms. Conclusions: The use of a DES to treat a previous DES in-stent restenosis represents a safe and efficient approach, with low rates of adverse events in the short and long terms.

DESCRIPTORS: Stents, adverse effects. Coronary restenosis. Angioplasty, transluminal, percutaneous coronary. Coronary disease. Treatment outcome.
O excelentes resultados clínicos obtidos com o uso de stents farmacológicos (SF) nos mais diversos cenários da prática intervencionista fizeram com que esses novos dispositivos ocupassem papel central no tratamento percutâneo da doença aterosclerótica coronária ${ }^{1-4}$. 
Entretanto, a despeito da marcante redução das taxas de reestenose obtidas com essas endopróteses, reobstrução coronária ainda ocorre em cerca de 5\% a $25 \%$ dos casos, dependendo da complexidade clínica e angiográfica da população tratada (por exemplo, diabéticos, vasos finos, lesões longas e bifurcações) $)^{5-7}$.

A presente análise tem por objetivo apresentar a experiência do Registro DESIRE no tratamento da reestenose após o implante de SF em pacientes não selecionados.

\section{MÉTODO}

\section{Casuística de desenho do estudo}

Desde maio de 2002, quando o primeiro SF foi aprovado para uso clínico em nosso País, todos os pacientes tratados exclusivamente com esses instrumentais no Hospital do Coração - Associação do Sanatório Sírio, em São Paulo, foram incluídos no Registro DESIRE (Drug-Eluting Stents $\underline{I} n$ the $\underline{R E a l}$ world). Os resultados gerais do DESIRE já foram previamente publicados $^{8,9}$, razão pela qual não nos deteremos pormenorizando o registro.

O presente estudo representa uma subanálise daquele registro, envolvendo todos os pacientes que apresentavam, como lesão-alvo, reestenose de um SF, implantado ou não em nossa Instituição, no período de maio de 2002 a novembro de 2007. Foram excluídos pacientes que apresentassem reestenose de enxertos venosos ou arteriais.

A reestenose do SF foi definida pela recorrência de sintomas isquêmicos e/ou exame não-invasivo indicativo de isquemia no território tratado, na presença de obstrução $\geq 50 \%$, à angiografia (avaliação visual), no interior do SF previamente implantado.

\section{Regime antitrombótico}

O protocolo antitrombótico consistiu na administração de dois agentes antiplaquetários: (1) ácido acetilsalicílico (AAS), na dose de 100-200 mg, iniciado pelo menos 24 horas antes do procedimento e prescrito indefinidamente; e (2) clopidogrel, na dose de ataque de 300-600 mg, também iniciado 24 horas antes da intervenção e mantido por um período mínimo de 12 meses.

Durante o procedimento, heparina foi administrada na dose de $70 \mathrm{UI} / \mathrm{kg}$ a $100 \mathrm{UI} / \mathrm{kg}$ e em doses adicionais, para manter o tempo de coagulação ativada > 250 segundos. A decisão sobre a utilização dos inibidores da glicoproteína IIb/IIla ficou a critério do operador.

\section{Intervenção coronária percutânea}

O implante de SF seguiu a técnica contemporânea estabelecida. Sempre que necessária, a pré-dilatação deveria ser realizada com balões curtos insuflados a baixas pressões, visando a evitar injúria nos segmentos adjacentes à lesão. O stent deveria ser implantado de forma a garantir a completa cobertura da lesão e, quando necessário, mais de um stent poderia ser realizado com a sobreposição das bordas dos mesmos.

Após o implante de stent, a pós-dilatação, quando indicada, deveria ser realizada com balões com extensão menor que a do stent implantado, com o cuidado de não ultrapassar suas bordas, evitando mais uma vez a injúria nos segmentos não cobertos pela prótese. Utilizamos os seguintes SF: stent Cypher ${ }^{\circledR}$ (Cordis, Johnson \& Johnson), com eluição de sirolimus, e stent Taxus $^{\circledR}$ (Boston Scientific Corporation), com eluição de paclitaxel. Esses stents estavam disponíveis em variações de extensão de $8 \mathrm{~mm}$ a $33 \mathrm{~mm}$ e diâmetros de 2,25 mm a 3,5 mm para o stent Cypher ${ }^{\circledR}$, e extensões de $8 \mathrm{~mm}$ a $32 \mathrm{~mm}$ e diâmetros de 2,25 mm a 4,5 mm para o stent Taxus $^{\circledR}$. A escolha do tipo de SF ficou a critério de cada operador.

A angiografia coronária quantitativa foi realizada imediatamente antes e após o implante do stent, por técnico treinado e experiente. A análise do segmento tratado compreendeu a área coberta pelo stent e as bordas $(5 \mathrm{~mm})$ proximal e distal. As mensurações angiográficas incluíram as referências proximal, distal e interpolada, o diâmetro mínimo da luz (DML), a porcentagem de obstrução e a extensão da lesão. Além disso, o ganho imediato foi avaliado a partir do cálculo da diferença entre o DML aferido imediatamente após e antes do implante do stent. Durante a hospitalização, todos os pacientes foram submetidos a avaliação com eletrocardiograma (ECG) e marcadores bioquímicos (CKMB), em três momentos: antes e imediatamente após a intervenção e na alta hospitalar.

\section{Objetivos do estudo e definições}

O desfecho primário deste estudo foi a avaliação da ocorrência de eventos cardíacos maiores no seguimento clínico tardio (> 6 meses). Os desfechos secundários incluíram as taxas de revascularização da lesão-alvo (RLA) e trombose do stent em todo o período do seguimento.

Os eventos cardíacos maiores foram definidos como óbito cardíaco, infarto do miocárdio e RLA. Os óbitos foram classificados como cardíacos e não-cardíacos. Óbitos de causas indeterminadas foram relatados como cardíacos. Os infartos do miocárdio foram definidos como o aparecimento de novas ondas $Q$ em duas derivações contíguas do ECG e/ou a elevação da CKMB $>3$ vezes o limite superior do normal. Todas as reintervenções, percutâneas ou cirúrgicas, resultantes da recorrência da obstrução (> 50\%) dentro do stent implantado no procedimento-índice ou no segmento arterial, que incluía os $5 \mathrm{~mm}$ proximais e os $5 \mathrm{~mm}$ distais ao stent, foram classificadas como RLA. A classificação proposta pelo Academic Research Consortium foi utilizada para a definição da trombose do stent 
como definitiva (confirmação angiográfica ou anatomopatológica), provável (morte súbita $\leq 30$ dias pósSF ou infarto relacionado à região da artéria tratada, mesmo sem confirmação angiográfica) e possível (morte súbita > 30 dias pós-stents farmacológicos). Quanto à distribuição temporal, as tromboses protéticas ainda foram divididas como: agudas e subagudas ( $\leq 30$ dias), tardias (31 dias a 360 dias) e muito tardias (> 360 dias). O sucesso angiográfico foi definido pela presença de lesão residual $<20 \%$ associada à presença de fluxo coronário TIMI 3 na ausência de trombos e/ou dissecções. O sucesso do procedimento foi considerado como o sucesso angiográfico na ausência de eventos cardíacos maiores.

A função ventricular esquerda foi avaliada pelo cálculo da fração de ejeção (\% FE) e classificada como: a) normal ( $\% \mathrm{FE} \geq 55 \%$ ); b) disfunção discreta (\% FE $\geq 40 \%<55 \%$; c) disfunção moderada ( $\%$ FE $\geq 30 \%$ $<40 \%$ ); e d) disfunção significativa (\% FE < 30\%).

A insuficiência renal crônica foi definida pela presença de valores séricos da creatinina $\geq 1,5 \mathrm{mg} / \mathrm{dl}$ e pela taxa de filtração glomerular (clearance) $<60 \mathrm{ml} /$ $\min / 1,73 \mathrm{~m}^{2}$.

O seguimento clínico foi obtido por visita médica ou por telefone, seguindo protocolo pré-definido, ao final de 30 dias, seis meses e um ano. A partir de então, o seguimento era feito anualmente.

\section{RESULTADOS}

Entre maio de 2002 e novembro de 2007, 61 $(2,9 \%)$ pacientes portadores de reestenose foram identificados (do total de 2.100 pacientes do DESIRE), excluindo-se desta análise 16 indivíduos por terem sido tratados por revascularização cirúrgica ou angioplastia com balão, restando, portanto, 45 pacientes neste estudo.

A Tabela 1 contém as principais características dos 45 pacientes incluídos neste estudo. A maioria era do sexo masculino $(66,7 \%)$, com média de idade de $59,6 \pm 9,5$ anos. Diabetes melito foi observado em $35,5 \%$ dessa coorte. A maior parte das reestenoses seguiu-se ao implante de SF para tratamento de lesões de novo $(88,9 \%)$, sendo a maioria do tipo focal $(<10 \mathrm{~mm}$ de extensão) e acometendo mais freqüentemente a artéria coronária direita $(41,5 \%)$.

A angiografia quantitativa pré-intervenção demonstrou que os vasos tratados tinham, em média, 2,99 \pm 0,5 mm de diâmetro e o porcentual de obstrução era de $66,8 \pm 11,4 \%$. Após a intervenção, a lesão residual foi de $5,04 \pm 2,41 \%$. Stent Cypher $^{\circledR}$ foi utilizado na maioria dos casos $(89,6 \%)$ e a troca por um SF diferente do que apresentou reestenose só ocorreu em 28\% dos casos. As taxas de sucesso angiográfico e clínico foram de $100 \%$ e $97,8 \%$, respectivamente. A Tabela 2 detalha o procedimento e apresenta os resultados da angiografia coronária quantitativa pré e pós-intervenção.
TABELA 1 Características clínicas e angiográficas de base

\begin{tabular}{lc}
\hline Características & $\mathbf{4 5}$ pacientes (53 lesões) \\
\hline Média de idade, anos & $59,6 \pm 9,5$ \\
Sexo feminino & $15(33,3 \%)$ \\
Diabetes melito & $16(35,5 \%)$ \\
HAS & $34(75,5 \%)$ \\
Dislipidemia & $37(82,2 \%)$ \\
Tabagismo & $12(26,6 \%)$ \\
História familiar de DAC & $20(44,4 \%)$ \\
Apresentação clínica & \\
$\quad$ Isquemia silenciosa/angina estável & $28(62,2 \%)$ \\
$\quad$ Angina instável/IAM sem supra ST & $17(27,8 \%)$ \\
Tipo de reestenose & \\
$\quad$ Focal & $40(75 \%)$ \\
$\quad$ Difusa & $13(15 \%)$ \\
Vaso tratado & $12(22,6 \%)$ \\
$\quad$ Artéria descendente anterior & $19(35,9 \%)$ \\
$\quad$ Artéria circunflexa & $22(41,5 \%)$ \\
$\quad$ Artéria coronária direita & $1(1,9 \%)$ \\
Uso de inibidor da GP IIb/IIla & \\
\hline
\end{tabular}

DAC = doença arterial coronária; GP = glicoproteína; HAS = hipertensão arterial sistêmica; IAM sem supra ST = infarto agudo do miocárdio sem supradesnivelamento do segmento ST.

Seguimento clínico tardio (3,6 \pm 1,2 anos) foi obtido em $97,8 \%$ dos pacientes. A Tabela 3 traz os resultados iniciais e tardios. Na fase hospitalar, um paciente evoluiu com elevação enzimática acima dos níveis considerados normais (infarto agudo do miocárdio nãoQ). No seguimento tardio, 5 pacientes apresentaram reestenose (focal) do SF implantado, totalizando 13,3\% de eventos cardíacos maiores acumulados. Todos esses pacientes voltaram a ser abordados percutaneamente com implante de outro SF. Notavelmente, não houve nenhum caso de trombose de stent nessa série.

\section{DISCUSSÃo}

O presente estudo confirma a hipótese de que o uso de um SF para tratar a reestenose de outro SF constitui-se em alternativa factível, segura e eficaz, com elevado sucesso imediato e baixa taxa de recorrência da lesão a longo prazo.

Por se tratar de um evento relativamente pouco freqüente, pouca informação está disponível sobre a melhor forma de se abordar a reestenose de SF.

Um dos primeiros estudos a avaliar o tratamento de reestenose de SF foi o de Lemos et al. ${ }^{10}$, comparando o emprego de outro SF à angioplastia apenas com cateter-balão e ao uso de stent não-farmacológico (SNF). A taxa geral de recorrência de reestenose foi de $42,9 \%$, sendo $29,3 \%$ no grupo que recebeu outro SF. Como 
TABELA 2

Análise angiográfica quantitativa

\begin{tabular}{lc}
\hline Características & $\mathbf{5 3}$ lesões/58 stents \\
\hline Extensão da lesão, mm & $11,33 \pm 5,8$ \\
Extensão do stent, mm & $18,93 \pm 5,47$ \\
Stent/paciente & 1,1 \\
Stent implantado & \\
$\quad$ Cypher & \\
$\quad$ Taxus & \\
Troca para um SF diferente do & $62(89,6 \%)$ \\
inicial (switch) & $6(11,4 \%)$ \\
Realização de pré-dilatação & $15(28 \%)$ \\
Realização de pós-dilatação & $11(20,7 \%)$ \\
Extensão do stent/extensão da lesão, mm & $28(53 \%)$ \\
Pressão máxima de liberação & $1,6 \pm 0,3$ \\
do stent, atm & $14,4 \pm 3,6$ \\
Diâmetro médio de referência & \\
do vaso, mm & \\
Diâmetro luminal mínimo, mm & $2,99 \pm 0,5$ \\
$\quad$ Pré-procedimento & \\
$\quad$ Pós-procedimento & $0,98 \pm 0,6$ \\
Diâmetro de estenose, $\%$ & $2,75 \pm 0,8$ \\
$\quad$ Pré-procedimento & \\
$\quad$ Pós-procedimento & $66,8 \pm 11,4$ \\
Ganho agudo, mm & $5,04 \pm 2,41$ \\
Estenose residual, \% & $1,85 \pm 0,5$ \\
Fluxo TIMl III & $5,04 \pm 2,41$ \\
Sucesso angiográfico & $100 \%$ \\
Sucesso do procedimento & $100 \%$ \\
\hline SF = stent farmacológico. & $97,8 \%$ \\
\hline
\end{tabular}

TABELA 3

Eventos cardíacos maiores nas fases hospitalar e tardia

\begin{tabular}{|c|c|}
\hline Eventos & $n=45$ \\
\hline \multicolumn{2}{|l|}{ Fase hospitalar } \\
\hline Óbito cardíaco & 0 \\
\hline IAM & $1(2,2 \%)^{*}$ \\
\hline RLA & 0 \\
\hline \multicolumn{2}{|c|}{ Tardio (3,6 \pm 1,2 anos) acumulado } \\
\hline Óbito cardíaco & 0 \\
\hline IAM & 1 \\
\hline RLA & $5(11,1 \%)$ \\
\hline ECAM combinados & $6(13,3 \%)$ \\
\hline \multicolumn{2}{|c|}{$\begin{array}{l}\text { * Infarto agudo do miocárdio sem supradesnivelamento do } \\
\text { segmento ST. } \\
\text { ECAM = eventos cardíacos adversos maiores; IAM = infarto } \\
\text { agudo do miocárdio; } n=\text { número de pacientes; RLA = } \\
\text { revascularização da lesão-alvo. }\end{array}$} \\
\hline
\end{tabular}

principais limitações desse estudo, cita-se o pequeno tamanho da amostra (24 pacientes) e a falta de randomização entre as estratégias de tratamento. A seguir, uma subanálise dos 22 pacientes incluídos no estudo SIRIUS tratados com SF e que apresentaram reestenose demonstrou que após o tratamento dessas reestenoses, em sua maioria focais, a taxa de recorrência ao final de um ano foi de $23 \%{ }^{11}$. Nesse estudo, também foram testadas, de forma não-randômica, diferentes abordagens para tratamento da reestenose, desde angioplastia com cateter-balão até braquiterapia.

Em estudo publicado em 2006, Torguson et al. ${ }^{12}$ compararam, de forma randomizada, a braquiterapia ao emprego de outro SF para tratamento de reestenose prévia de SF. Ambos os métodos demonstraram-se seguros e eficazes em reduzir a incidência de nova reestenose a curto e médio prazos (seguimento até um ano). Entretanto, questões relacionadas à segurança a longo prazo da braquiterapia (trombose tardia e muito tardia e potencial combinação dos efeitos adversos da radiação e do fármaco componente do SF) bem como indícios prévios de ausência de manutenção dos resultados a longo prazo (late catch-up), aliados à elevada complexidade na execução dos procedimentos, têm progressivamente reduzido o entusiasmo por essa abordagem em todo o mundo ${ }^{13,14}$.

Nesse contexto, o implante de outro SF pode ser uma alternativa preferencial, dada a facilidade e a segurança dessa estratégia. Várias séries contemporâneas, em geral com pequeno número de pacientes incluídos, têm confirmado não só a exeqüibilidade dessa abordagem, como também os excelentes resultados com a utilização dos stents Cypher $^{\circledR}$ e Taxus $^{\circledR}$.

No entanto, em nossa opinião, independentemente da abordagem adotada no tratamento da reestenose de SF, o conhecimento do mecanismo que levou a sua ocorrência e, sobretudo, sua apresentação angiográfica (focal vs. difusa) representam os elementos fundamentais no sucesso da nova intervenção.

Ainda que descrita em modelos experimentais ${ }^{15}$, a falência do fármaco antiproliferativo parece ocupar papel secundário na etiologia dessas reestenoses no cenário clínico, sendo predominantes nesse cenário as causas mecânicas (má-expansão da endoprótese, cobertura incompleta da lesão, distribuição não-uniforme das hastes do stent, etc. $)^{16-19}$. Contra a hipótese de falência do fármaco empregado como causa de reestenose, Garg et al. ${ }^{20}$ publicaram os resultados de uma série consecutiva de 116 pacientes tratados com SF e que evoluíram com reestenose no Washington Hospital Center, notando que o implante de um SF diferente daquele que apresentou reobstrução apresentava resultados similares ao emprego de SF igual ao anteriormente implantado (eventos cardíacos maiores de $14,5 \%$ vs. $16,7 \%, p=0,75$; taxa de reestenose de $32,6 \%$ vs. $35 \%$, p = 0,81). Nesse contexto, a utilização de ultra-som intracoronário poderia exercer papel 
relevante na identificação do mecanismo de reestenose e mesmo guiar o implante do novo SF. Entretanto, questões relacionadas ao custo e à aprovação dessa técnica pelas seguradoras de saúde dificultam seu emprego em nosso meio.

Na presente análise, praticamente $90 \%$ das reestenoses eram do tipo focal, possivelmente refletindo o predomínio da utilização do stent Cypher ${ }^{\circledR}$ nesse registro. Trabalhos prévios demonstraram que o uso do stent Taxus $^{\circledR}$ está mais relacionado à apresentação difusa da reestenose ${ }^{21}$.

Por fim, um fato que merece destaque é a ausência de casos de trombose nesta série, apesar da presença de múltiplas camadas de stents e polímeros e, em alguns casos, da sobreposição de fármacos diferentes. De modo geral, a ocorrência de trombose nesse subgrupo de pacientes tem sido baixa nas séries publicadas com emprego de outro SF, estando abaixo do reportado para as populações gerais tratadas com SF, o que fala a favor da segurança do procedimento.

\section{Limitações}

O presente estudo é retrospectivo e, portanto, possui todas as limitações inerentes ao modelo. Além disso, o baixo número de pacientes incluídos, embora reflita a realidade da reestenose na prática clínica na era dos SF, pode ter diminuído o poder desta análise em detectar eventos adversos. A falta de um grupo controle constituído por pacientes tratados com terapêuticas alternativas (angioplastia com cateter-balão, braquiterapia, etc.) impossibilita comparar as diferentes modalidades terapêuticas nesse complexo cenário. Finalmente, a ausência de randomização para definir quem seria tratado com o mesmo tipo de SF ou quem receberia um SF diferente daquele que apresentou reestenose impossibilita estimar a efetividade dessa opção terapêutica no tratamento da reestenose de SF.

\section{CONCLUSÕES}

Em nossa experiência com pacientes não-selecionados do Registro DESIRE, o uso de SF para tratar a reestenose de SF previamente implantados mostrou ser uma opção terapêutica factível, simples e segura, com baixas taxas de eventos adversos a curto e longo prazos. Entretanto, ainda há espaço para se desenvolver novas opções terapêuticas, visando à redução da recorrência da reestenose nessa complexa população. Destacam-se, como opções promissoras, o reimplante de SF guiado por ultra-som intracoronário e o uso das novas gerações de SF.

\section{REFERÊNCIAS BIBLIOGRÁFICAS}

1. Morice MC, Serruys PW, Sousa JE, Fajadet J, Ban Hayashi E, Perin M. Randomized Study with the Sirolimus-Coated Bx Velocity Balloon-Expandable Stent in the Treatment of Patients with de Novo Native Coronary Artery Lesions. A randomized comparison of a sirolimus-eluting stent with a standard stent for coronary revascularization. RAVEL Study Group. N Engl J Med. 2002;346(23):1773-80.

2. Moses JW, Leon MB, Popma JJ, Fitzgerald PJ, Holmes DR, O'Shaughnessy C, et al. Sirolimus-eluting stents versus standard stents in patients with stenosis in a native coronary artery. SIRIUS Investigators. N Engl J Med. 2003;349(14):1315-23.

3. Stone GW, Ellis SG, Cox DA, Hermiller J, O'Shaughnessy C, Mann JT, et al. A polymer-based, paclitaxel-eluting stent in patients with coronary artery disease. TAXUS-IV Investigators. N Engl J Med. 2004;350(3):221-31.

4. Sousa JE, Costa MA, Tuzcu EM, Yadav JS, Ellis S. New frontiers in interventional cardiology. Circulation. 2005;111(5): 671-81.

5. Colombo A, Moses JW, Morice MC, Ludwig J, Holmes DR Jr, Spanos V, et al. Randomized study to evaluate sirolimuseluting stents implanted at coronary bifurcation lesions. Circulation. 2004;109(10):1244-9.

6. Mahmud E, Bromberg-Marin G, Palakodeti V, Ang L, Creanga D, Demaria AN. Clinical efficacy of drug-eluting stents in diabetic patients: a meta-analysis. J Am Coll Cardiol. 2008;51 (25):2385-95.

7. Elezi S, Dibra A, Mehilli J, Pache J, Wessely R, Schömig A, et al. Vessel size and outcome after coronary drug-eluting stent placement: results from a large cohort of patients treated with sirolimus- or paclitaxel-eluting stents. J Am Coll Cardiol. 2006;48(7):1304-9.

8. Sousa AGMR, Costa Jr JR, Moreira A, Costa RA, Cano MN, Andrade GAM, et al. Evolução clínica tardia dos stents farmacológicos: segurança e eficácia até cinco anos do registro DESIRE. Rev Bras Cardiol Invas. 2007;15(3):221-7.

9. Sousa A, Costa Jr JR, Moreira AC, Cano M, Maldonado G, Costa RA, et al. Long-term clinical outcomes of the DrugEluting Stents In the REal world (DESIRE) Registry. J Interv Cardiol. 2008;21(4):307-14.

10. Lemos PA, Saia F, Ligthart JM, Arampatzis CA, Sianos G, Tanabe $\mathrm{K}$, et al. Coronary restenosis after sirolimus-eluting stent implantation: morphological description and mechanistic analysis from a consecutive series of cases. Circulation. 2003;108(3):257-60.

11. Moussa ID, Moses JW, Kuntz RE, Holmes DR, Popma JJ, Teirstein PS, et al. The fate of patients with clinical recurrence after sirolimus-eluting stent implantation (a two-year follow-up analysis from the SIRIUS trial). Am J Cardiol. 2006;97(11):1582-4.

12. Torguson R, Sabate M, Deible R, Smith K, Chu WW, Kent $\mathrm{KM}$, et al. Intravascular brachytherapy versus drug-eluting stent for the treatment of patients with drug-eluting stent restenosis. Am J Cardiol. 2006;98(10):1340-4.

13. Costa MA, Sabaté M, van der Giessen WJ, Kay IP, Cervinka $\mathrm{P}$, Ligthart JM, et al. Late coronary occlusion after intracoronary brachytherapy. Circulation. 1999;100(8):789-92.

14. Feres F, Munoz J, Abizaid A, Staico R, Kuwabara M, Mattos $\mathrm{L}$, et al. Angiographic and intravascular ultrasound findings of the late catch-up phenomenon after intracoronary betaradiation for the treatment of in-stent restenosis. J Invasive Cardiol. $2005 ; 17(9): 473-7$.

15. Zhang Q, Lu L, Pu L, Zhang R, Shen J, Zhu Z, et al. Neointimal hyperplasia persists at six months after sirolimuseluting stent implantation in diabetic porcine. Cardiovasc Diabetol. 2007;6:16.

16. Fujii K, Mintz GS, Kobayashi Y, Carlier SG, Takebayashi H, Yasuda T, et al. Contribution of stent underexpansion to recurrence after sirolimus-eluting stent implantation for instent restenosis. Circulation. 20049;109(9):1085-8.

17. Costa MA, Angiolillo DJ, Tannenbaum M, Driesman M, 
Costa Jr. JR, et al. Implante de Stent Farmacológico para o Tratamento da Reestenose de Outro Stent Farmacológico: Análise Tardia do Registro DESIRE. Rev Bras Cardiol Invas. 2008;16(3):273-278.

Chu A, Patterson J, et al. Impact of stent deployment procedural factors on long-term effectiveness and safety of sirolimus-eluting stents (final results of the multicenter prospective STLLR trial). STLLR Investigators. Am J Cardiol. 2008;101(12):1704-11.

18. Takebayashi H, Mintz GS, Carlier SG, Kobayashi Y, Fujii K, Yasuda $T$, et al. Nonuniform strut distribution correlates with more neointimal hyperplasia after sirolimus-eluting stent implantation. Circulation. 2004;110(22):3430-4.

19. Kim SW, Mintz GS, Lee KJ, Pregowski J, Tyczynski P, Escolar E, et al. Repeated stenting of recurrent in-stent restenotic lesions: intravascular ultrasound analysis and clinical outcome. J Invasive Cardiol. 2007;19(12):506-9.

20. Garg S, Smith K, Torguson R, Okabe T, Slottow TL, Steinberg $\mathrm{DH}$, et al. Treatment of drug-eluting stent restenosis with the same versus different drug-eluting stent. Catheter Cardiovasc Interv. 2007;70(1):9-14.

21. Solinas E, Dangas G, Kirtane AJ, Lansky AJ, Franklin-Bond $\mathrm{T}$, Boland $\mathrm{P}$, et al. Angiographic patterns of drug-eluting stent restenosis and one-year outcomes after treatment with repeated percutaneous coronary intervention. Am J Cardiol. $2008 ; 102(3): 311-5$. 